\title{
Thioredoxin down-regulation in the cytosol in thioredoxin 2 transgenic mice did not have beneficial effects to extend lifespan in male C57BL/6 mice
}

\author{
Madeline G. Roman ${ }^{a}$, Lisa C. Flores ${ }^{a}$, Geneva M. Cunningham ${ }^{a}$, Christie Cheng ${ }^{a}$, Colton Allen ${ }^{a}$, \\ Yidong Bai ${ }^{\mathrm{c}}$, Gene B. Hubbard ${ }^{\mathrm{a}, \mathrm{b}}$, Yuji Ikeno ${ }^{\mathrm{a}, \mathrm{b}, \mathrm{d},{ }^{*}}$
}

\author{
${ }^{a}$ Barshop Institute for Longevity and Aging Studies, The University of Texas Health Science Center at San Antonio, San \\ Antonio, TX 78229, USA. \\ ${ }^{b}$ Department of Pathology, The University of Texas Health Science Center at San Antonio, San Antonio, TX 78229, USA. \\ ${ }^{c}$ Department of Cell Systems and Anatomy, The University of Texas Health Science Center at San Antonio, San Antonio, \\ TX 78229, USA. \\ ${ }^{d}$ Geriatric Research Education and Clinical Center (GRECC), Audie L. Murphy VA Hospital, South Texas Veterans Health \\ Care System, San Antonio, TX 78229, USA.
}

\begin{abstract}
Background: This study was conducted to test the effects of thioredoxin (Trx)1 down-regulation in Trx2 transgenic $\left[\operatorname{Tg}(T X N 2)^{+/ 0}\right]$ mice on lifespan and age-related diseases. Our previous study with $\operatorname{Tg}(T X N 2)^{+/ 0}$ mice showed that mitochondrial Trx overexpression produced minimal life-extending effects with a slightly elevated severity of lymphoma and another study with a limited number of Trx1KO mice showed that there was a slight reduction of neoplastic lesions. Thus, this study was aimed to test if reduced Trx1 expression in combination with elevated Trx2 has beneficial effects on lifespan in mice by attenuating age-related diseases, specifically cancer.

Methods: Trx2 hemizygous transgenic and Trx1 heterozygous knockout mice $\left[\operatorname{Tg}(T X N 2)^{+/ 0} \mathrm{x} \operatorname{Trx} 1 \mathrm{KO}\right]$ were generated for survival and cross-sectional pathology experiments.

Results: $\operatorname{Tg}\left(T_{X N 2}\right)^{+/ 0}$ x Trx1KO mice showed significantly higher (approximately 1.5- to 3-fold) Trx2 levels and significantly less (approximately 50\% less) Trx1 levels in all of the tissues we examined compared to wildtype (WT) littermates. Trx1 down-regulation along with Trx2 overexpression did not change the levels of glutathione or other major antioxidant enzymes. Male $\operatorname{Tg}(T X N 2)^{+/ 0}$ x Trx1KO mice demonstrated only a slight extension of lifespan in the early part of life and no significant effects on the later part of life were observed, which was similar to our previous study with $\operatorname{Tg}(T X N 2)^{+/ 0}$ mice. $\operatorname{Tg}(T X N 2)^{+/ 0}$ x $\operatorname{Trx} 1 \mathrm{KO}$ mice had similar tumor burden, disease burden, incidence and severity of lymphoma, and severity of glomerulonephritis compared to WT mice at 22-26 months.

Conclusions: Our findings suggest that the combined Trx down-regulation in cytosol along with upregulation in mitochondria of $\operatorname{Tg}(T X N 2)^{+/ 0}$ x Trx1KO mice did not provide beneficial effects on aging, i.e., extend the lifespan or reduce age-related pathology compared to WT mice.
\end{abstract}

Keywords: Thioredoxin, transgenic mouse, knockout mouse, aging, cancer

\section{Introduction}

Possible beneficial roles of thioredoxin (Trx) in aging and

\footnotetext{
* Corresponding author: Yuji Ikeno

Mailing address: Barshop Institute for Longevity and Aging Studies, The University of Texas Health Science Center at San Antonio, 4939 Charles Katz Dr, San Antonio, TX 78229, USA. Email: ikeno@uthscsa.edu
}

Received: 22 November 2020 / Accepted: 20 December 2020 age-related diseases have been investigated in multiple studies, including several performed in our laboratory [1-5]. Trx is of interest in aging research because of its unique ability to alter oxidative stress and redox state, thereby affecting redox-sensitive signaling and subsequent effects on pathophysiology. This small protein $(12 \mathrm{kDa})$ has two redox-active cysteine residues in the active center (Cys-Gly-Pro-Cys). It plays an important role as the reductant for various molecules via thiol-disulfide exchange reactions [6-12]. These rapid and readily reversible reactions are ideal to control protein function by changing the redox state of structural or catalytic SH groups of proteins. 
Our laboratory has conducted aging studies with mice overexpressing or downregulating Trx in the cytosol and/ or mitochondria to test the effects of Trx on aging and age-related diseases [2-5]. First, we examined the effects of Trx1 (cytosolic Trx) overexpression on aging with two lines of transgenic mice $\left[\operatorname{Tg}(\operatorname{act}-T X N)^{+/ 0}\right.$ and $\operatorname{Tg}(T X N)^{+/ 0}$ mice] $[2,3]$. Survival experiments with these mice showed that overexpression of Trx 1 slightly extended the earlier part of life but did not show significant effects on maximum lifespans. Interestingly, Trx1 overexpression was accompanied by accelerated cancer development in the later part of life $[2,3]$.

These initial studies with Trx 1 transgenic mice led us to conduct another survival study with mice overexpressing Trx in mitochondria ( $\operatorname{Tr} x 2)$ because the importance of antioxidant overexpression in mitochondria in aging was strongly suggested by the study by Schriner et al [13]. The survival study using $\operatorname{Trx} 2$ transgenic mice $\left[\operatorname{Tg}(T X N 2)^{+/ 0}\right]$ showed that Trx overexpression in mitochondria had a slight but not significant extension (approximately 8-9\%) of mean, median, and 10th percentile lifespans compared to WT mice. $\operatorname{Tg}(T X N 2)^{+/ 0}$ mice also showed a slightly higher severity of lymphoma compared to WT mice [4].

Because we have not observed significant effects on lifespan, specifically in the latter part of life, by Trx overexpression in cytosol or mitochondria alone, we further tested if it is necessary to have changes in the levels of Trx in both the cytosol and mitochondria. One of the potential reasons Trx 2 overexpression did not significantly extend lifespan could be due to its effects on age-related tumor formation [4]. Both Trx 1 and Trx 2 transgenic mice showed a tendency to accelerate tumor development with age [2-4]. Our previous study with Trx1KO mice showed that reduced Trx1 did not change the lifespan, i.e., no extension or shortening; however, there was a subtle reduction of age-related cancer based on limited cross-sectional pathological analyses [5]. These observations led us to the hypothesis that reducing Trx 1 could prevent age-related tumor development in $\operatorname{Tg}(T X N 2)^{+/ 0}$ mice, resulting in an exteded lifespan compared to WT mice.

Thus, the purpose of this study is to examine the effects of reduced Trx 1 along with Trx 2 overexpression on aging and age-related diseases. We conducted a survival study using mice overexpressing Trx 2 and down-regulating $\operatorname{Trx} 1\left[\operatorname{Tg}(T X N 2)^{+/ 0} \times \operatorname{Trx} 1 \mathrm{KO}\right.$ mice $]$. We report that the combined Trx down-regulation in cytosol and upregulation in mitochondria did not extend the lifespan, although these mice did show a slight extension (approximately $30.7 \%$ ) of lifespan in the early stage of life. Age-related pathology in $\operatorname{Tg}(T X N 2)^{+/ 0} \mathrm{x}$ Trx1KO mice was similar to WT mice. Therefore, our results suggest that reduced Trx1 along with Trx 2 overexpression did not have significant effects on lifespan or age-related diseases including tumor development in male C57BL/6 mice.

\section{Materials and Methods}

\section{Animals and animal husbandry}

$\operatorname{Tg}(T X N 2)^{+/ 0}$ mice were generated using the human thioredoxin 2 gene [a PAC clone (RP5-1119A7), Children's Hospital Oakland Research Institute's (CHORI) BACPAC Resources Center (BPRC), Oakland, CA] as previously described [4]. Trx 1 heterozygous knockout mice were generated with an ES clone purchased from Lexicon Pharmaceuticals, Inc. (OST452454) as previously described [5]. Male heterozygous $\operatorname{Trx} 1 \mathrm{KO}$ mice were crossed to female hemizygous Trx 2 transgenic mice $\left[\operatorname{Tg}(T X N 2)^{+/ 0}\right]$ to generate $\operatorname{Tg}(T X N 2)^{+/ 0} \times \operatorname{Trx} 1 \mathrm{KO}$ and WT control mice. These mice were fed ad libitum with a commercial chow (Teklad Diet LM485: Madison, WI) and free access to acidified $(\mathrm{pH}=2.6-2.7)$ filtered reverse osmosis water. The amount of chow removed from the cage hopper and the spillage (the chow on the bottom of the cage) were weighed monthly to accurately measure the food consumption. All of the mice were weighed monthly. The mice were maintained pathogen-free in microisolator units on Tek FRESH ${ }^{\circledR}$ ultra laboratory bedding. Sentinel mice housed in the same room and exposed weekly to bedding collected from the cages of experimental mice were sacrificed on receipt and every six months thereafter for monitoring of viral antibodies (Mouse Level II Complete Antibody Profile CARB, Ectro, EDIM, GDVII, LCM, M. Ad-FL, M. Ad-K87, MCMV, MHV, M. pul., MPV, MVM, Polyoma, PVM, Reo, Sendai; BioReliance, Rockville, MD). All tests were negative.

\section{Determination of $\operatorname{Tr} 22$ expression}

The mitochondrial fraction obtained from several tissues (liver, kidney, brain) from young (4-6 months old) $\operatorname{Tg}(T X N 2)^{+/ 0} \times$ Trx1KO and WT mice were used to measure thioredoxin 2 (Trx2) levels by Western blot analysis with rabbit anti-Trx2 polyclonal antibody (Catalog No. LF-PA0012; LabFrontier, Seoul, South Korea) [4, 14]. After incubation with the primary antibodies, membranes were incubated with the respective peroxidase-linked secondary antibodies (Catalog No. P0217; Dako, Carpinteria, CA). Chemiluminescence was detected using the ECL Western blot detection kit (Amersham Biosciences Corp., Piscataway, NJ).

\section{Trx1 levels}

Trx1 levels were measured using cytosolic fractions obtained from tissues (liver, kidney, brain) of young (4-6 months old) $\operatorname{Tg}(T X N 2)^{+/ 0}$ x Trx1KO and WT mice. Western blot analysis using goat anti-human Trx1 polyclonal antibodies (Catalog No. 705; American Diagnostica, Inc., Greenwich, CT) was performed as previously described [2, 3]. Total Trx1 (both oxidized and reduced forms) levels were measured by these antibodies. After incubation with the primary antibody, membranes were incubated with the peroxidase-linked secondary antibody (Catalog No. P0449; Dako, Carpinteria, CA). Chemiluminescence was detected with an ECL Western blot detection kit (Amersham Biosciences Corp., Piscataway, NJ). 


\section{Total glutathione levels}

The Bioxytech GSH-420 kit (Catalog No. 21023; Oxis International, Inc., Foster City, CA) was used to measure the levels of total glutathione in several tissues (liver, kidney) from young (4-6 months old) $\operatorname{Tg}(T X N 2)^{+/ 0} \mathrm{x} \operatorname{Trx} 1 \mathrm{KO}$ and WT mice.

Determination of major antioxidant enzyme activities: Cu/ZnSOD, MnSOD, glutathione peroxidase, and catalase

Major antioxidant enzymes [Cu/ZnSOD, MnSOD, glutathione peroxidase (GPx), and catalase] activities were measured in tissue homogenates obtained from the liver and kidney of young (4-6 months old) $\operatorname{Tg}(T X N 2)^{+/ 0} \mathrm{x}$ Trx1KO and WT mice. The antioxidant enzymatic activity assays were conducted with the supernatants. GPx activity in tissue homogenates was measured by the assay as previously described [15]. The Catalase-520TM assay kit (OxisResearchTM, Portland, OR) was used to measure catalase activity by measuring the decomposition of hydrogen peroxide at $520 \mathrm{~nm}$. MnSOD and $\mathrm{Cu} / \mathrm{ZnSOD}$ levels were determined using activity gels $[16,17]$. Images of the gels were analyzed by ImageQuant software.

\section{Survival study}

Mice in the survival groups were allowed to live out their lives, and the lifespan for individual mice was determined by recording the age of spontaneous death. A survival study was conducted with $35 \operatorname{Tg}(T X N 2)^{+/ 0} \mathrm{x} \operatorname{Trx} 1 \mathrm{KO}$ and 35 WT male mice. The survival curves comparison was statistically analyzed by the log-rank test [18]. The data for mean, 75th percentile, median, and 10th percentile (when $90 \%$ of the mice had died) survival were calculated for $\operatorname{Tg}(T X N 2)^{+/ 0} \times$ Trx1KO and WT mice. The mean survivals for $\operatorname{Tg}(T X N 2)^{+/ 0} \mathrm{x} \operatorname{Trx} 1 \mathrm{KO}$ and WT male mice were compared by performing a Student's $t$-test upon log-transformed survival times. The median and $10^{\text {th }}$ percentile survivals for $\operatorname{Tg}(T X N 2)^{+/ 0} \times \operatorname{Trx} 1 \mathrm{KO}$ and WT mice were compared using a score test adapted from Wang et al. [19].

\section{Cross-sectional pathological assessment}

$\operatorname{Tg}(T X N 2)^{+/ 0} \times \operatorname{Trx} 1 \mathrm{KO}(n=21)$ and WT $(n=40)$ male mice were assigned to the cross-sectional pathological analyses at 22-26 months of age. The gross pathological examinations to detect the visible tumors and other agerelated lesions were conducted with each animal. Then, the following organs and tissues were excised and preserved in $10 \%$ buffered formalin: brain, pituitary gland, heart, lung, trachea, thymus, aorta, esophagus, stomach, small intestine, colon, liver, pancreas, spleen, kidneys, urinary bladder, reproductive system (prostate, testes, epididymis, and seminal vesicles), thyroid gland, adrenal glands, parathyroid glands, psoas muscle, knee joint, sternum, and vertebrae. Any other tissues with gross lesions were also excised. After the fixation, tissues were processed conventionally, embedded in paraffin, sectioned at $5 \mu \mathrm{m}$, and stained with hematoxylin-eosin. Histological classifications in aging mice were conducted after the diagnosis of each histopathological change was made [20, 21]. A list of pathological lesions for both neoplastic and non-neoplastic diseases was constructed for each mouse. These histopathological data were utilized to determine the tumor burden, disease burden, and severity of each lesion in each mouse [1-4, 21-23].

\section{Statistical analysis}

Unless otherwise specified, all experiments were done at least in triplicate. Data were expressed as means \pm SEM and were analyzed by the non-parametric test ANOVA. All pair-wise contrasts were computed using Tukey error protection at $95 \%$ CI unless otherwise indicated. Differences were considered statistically significant at $P<0.05$.

\section{Results}

\section{Levels of $\operatorname{Trx} 1$ and $\operatorname{Trx} 2$ in tissues from $\operatorname{Tg}(T X N 2)^{+/ 0} x$ Trx1KO mice}

The levels of Trx 1 and Trx 2 in tissues (liver, kidney, brain) from young (4-6 months old) $\operatorname{Tg}(T X N 2)^{+/ 0} \mathrm{x}$ Trx1KO and WT mice were measured using Western blot analysis. The Trx 2 protein levels were significantly higher (approximately 1.3 - to 3 -fold) in all three tissues examined in young $\operatorname{Tg}(T X N 2)^{+/ 0} \mathrm{x} \operatorname{Tr} \times 1 \mathrm{KO}$ mice compared to

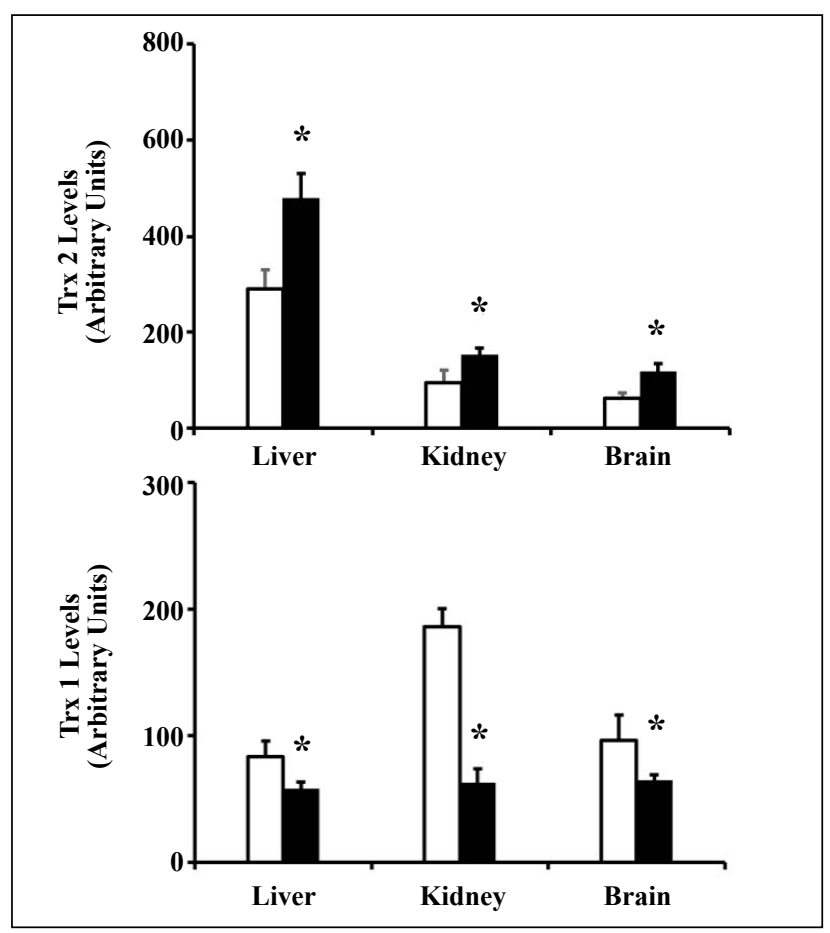

Figure 1. Trx2 and Trx1 levels in young $\operatorname{Tg}(T X N 2)^{+/ 0} \times \operatorname{Trx} 1 \mathrm{KO}$ mice and their WT littermates. Trx 2 and Trx 1 protein levels were determined by Western blot in various tissues of 4 to 6 month old $\operatorname{Tg}(T X N 2)^{+/ 0} \times \operatorname{Tr} x 1 \mathrm{KO}$ and WT mice. Trx 2 level was significantly (1.53 fold) higher in the tissues (liver, kidney, and brain) of 4 to 6 month old $\operatorname{Tg}(T X N 2)^{+/ 0} \times \operatorname{Trx} 1 \mathrm{KO}$ (closed bars) and WT mice (open bars) (A, * $P$ $<0.05$ ). Trx 1 was significantly lower (approximately $50 \%$ less) in the tissues (liver, kidney, and brain) of $\operatorname{Tg}(T X N 2)^{+/ 0} \times \operatorname{Trx} 1 \mathrm{KO}$ (closed bars) and WT littermates (open bars) $(\mathbf{B}, * P<0.05)$. The data are the mean SEM from three to five mice. 
their WT littermates (Figure 1A; $P<0.05$ ). Levels of Trx1 were significantly lower (approximately 50\% less) in all three tissues examined in young $\operatorname{Tg}(T X N 2)^{+/ 0} \mathrm{x} \operatorname{Trx} 1 \mathrm{KO}$ mice compared to their WT littermates (Figure 1B; $P<$ $0.05)$.

\section{Levels of total glutathione and major antioxidant en- zymes in tissues from $\operatorname{Tg}(T X N 2)^{+/ 0} \times \operatorname{Trx} 1 \mathrm{KO}$ mice}

To examine whether the reduced Trx 1 along with increased levels of Trx 2 affects the levels of glutathione, which has similar biological functions to Trx, we measured total glutathione levels in liver and kidney from young (4-6 months old) $\operatorname{Tg}(T X N 2)^{+/ 0} \mathrm{x} \operatorname{Trx} 1 \mathrm{KO}$ and WT mice. The data in Figure 2 show that levels of total glutathione in the tissues (liver, kidney) were similar between $\operatorname{Tg}(T X N 2)^{+/ 0} \mathrm{x}$ Trx1KO and WT mice at 4-6 months (Figure $2 ; P>0.05)$.

$\operatorname{Tg}(T X N 2)^{+/ 0}$ x Trx1KO and WT mice showed similar activity/levels for major antioxidant enzymes $(\mathrm{Cu} / \mathrm{ZnSOD}$, MnSOD, GPx, and catalase) in the liver and kidney at 4-6 months (Figure 3; $P>0.05$ ).

\section{Survival curves, body and organ weights, and food consumption}

The survival study was conducted with male $\operatorname{Tg}(T X N 2)^{+/ 0}$ $\mathrm{x} \operatorname{Trx} 1 \mathrm{KO}(n=35)$ and WT $(n=35)$ mice (Figure 4$)$. The survival curve of $\operatorname{Tg}(T X N 2)^{+/ 0} \mathrm{x} \operatorname{Tr} \times 1 \mathrm{KO}$ mice was not significantly different from WT mice (Log-rank: $P=0.45$ ). The mean, 75th percentile, median, and 10th percentile survival for 1) WT mice were 746, 470, 763, and 1,117 days; and 2) $\operatorname{Tg}(T X N 2)^{+/ 0} \times \operatorname{Trx} 1 \mathrm{KO}$ mice were 835,678 , 791 , and 1,141 days, respectively (Figure 4). $\operatorname{Tg}(T X N 2)^{+/ 0}$ $\mathrm{x}$ Trx $1 \mathrm{KO}$ mice had slightly longer mean (10.7), 75th percentile $(30.7 \%)$, median $(3.5 \%)$, and 10th percentile (2.1\%) lifespans compared to WT mice, although these differences were not statistically significant $(P>0.05)$. $\operatorname{Tg}(T X N 2)^{+/ 0} \times$ Trx1KO mice had similar body and organ weights to WT mice at 4-6 months of age (Table 1). $\operatorname{Tg}(T X N 2)^{+/ 0} \mathrm{x}$ Trx1KO and WT mice had similar food intake (data not shown).

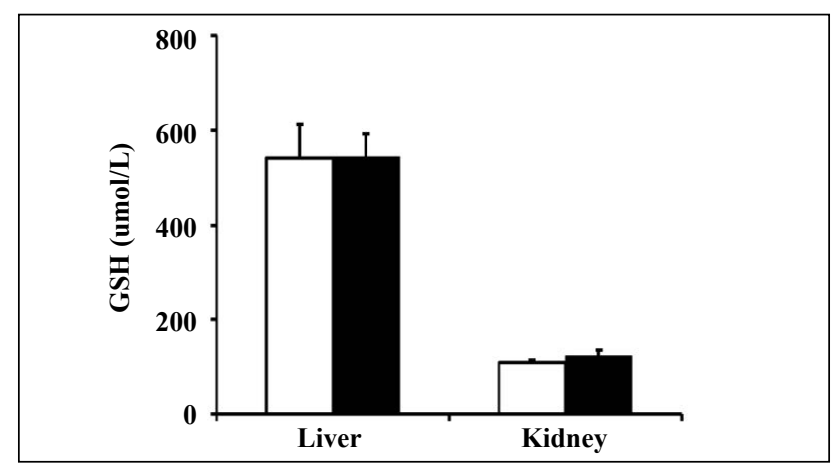

Figure 2. Levels of total glutathione in $\operatorname{Tg}(T X N 2)^{+/ 0} \times \operatorname{Trx} 1 \mathrm{KO}$ and WT mice. Total glutathione levels were measured in the liver and kidney of young 4 to 6 month $\operatorname{Tg}(T X N 2)^{+/ 0} \times$ Trx $1 \mathrm{KO}$ mice (closed bar) and WT mice (open bar). No significant difference was observed in total glutathione levels in $\operatorname{Tg}(T X N 2)^{+/ 0} \times \operatorname{Trx} 1 \mathrm{KO}$ compared to WT mice. The data are the mean SEM from three to five mice.
Table 1. Body and Organ Weights of 4-6M Tg(TXN2) ${ }^{+/ 0} \mathrm{x} \operatorname{Trx} 1 \mathrm{KO}$ mice.

\begin{tabular}{lcc}
\hline & $\begin{array}{c}\text { WT } \\
(n=3)\end{array}$ & $\begin{array}{c}\text { Tg(TXN2) } \\
(n=4)\end{array}$ \\
\hline Body Weight (g) & $29.214 \pm 2.14$ & $27.279 \pm 1.695$ \\
Liver (g) & $1.474 \pm 0.069$ & $1.447 \pm 0.069$ \\
Spleen (g) & $0.168 \pm 0.012$ & $0.165 \pm 0.012$ \\
Pancreas (g) & $0.287 \pm 0.035$ & $0.180 \pm 0.019$ \\
Heart (g) & $0.090 \pm 0.017$ & $0.085 \pm 0.012$ \\
Lung (g) & $0.189 \pm 0.026$ & $0.182 \pm 0.006$ \\
Left Kidney (g) & $0.229 \pm 0.024$ & $0.216 \pm 0.013$ \\
Right Kidney (g) & $0.246 \pm 0.024$ & $0.245 \pm 0.009$ \\
Left Testicle (g) & $0.130 \pm 0.011$ & $0.115 \pm 0.008$ \\
Right Testicle (g) & $0.127 \pm 0.007$ & $0.115 \pm 0.011$ \\
Brain (g) & $0.458 \pm 0.015$ & $0.455 \pm 0.011$ \\
\hline
\end{tabular}

\section{Cross-sectional pathology}

The cross-sectional pathological analyses were conducted with $21 \mathrm{Tg}(T X N 2)^{+/ 0} \mathrm{x}$ Trx1KO mice and $40 \mathrm{WT}$ mice (22-26 months old). The major age-related pathology was a neoplastic disease, and commonly observed tumors were lymphoma, hemangioma/hemangiosarcoma (liver and spleen), adenocarcinoma (lung), and hepatocellular carcinoma (liver), which is consistent with previous pathology results from the mice that have C57BL/6 genetic background $[1-4,21]$.

The tumor burden, which is the total number of different types of tumors for each mouse, in both $\operatorname{Tg}(T X N 2)^{+/ 0} \mathrm{x}$ Trx1KO and WT groups is presented in Figure 5A. The tumor burden of $\operatorname{Tg}(T X N 2)^{+/ 0} \times \operatorname{Trx} 1 \mathrm{KO}$ mice was similar to WT mice $(P>0.05)$. The incidence of lymphoma was $9 \%$ less in $\operatorname{Tg}(T X N 2)^{+/ 0} \times \operatorname{Trx} 1 \mathrm{KO}(76 \%)$ than WT $(85 \%)$ mice, and severity of lymphoma was also similar between $\operatorname{Tg}(T X N 2)^{+/ 0} \mathrm{x} \operatorname{Trx} 1 \mathrm{KO}(2.50)$ and WT (2.74) mice (Figure 5B; $P>0.05)$.

We also compared the severity of glomerulonephritis, a major non-neoplastic disease, in $\operatorname{Tg}(T X N 2)^{+/ 0} \mathrm{x} \operatorname{Trx} 1 \mathrm{KO}$ and WT mice. The severity of glomerulonephritis was slightly higher in $\operatorname{Tg}(T X N 2)^{+/ 0} \mathrm{x} \operatorname{Trx} 1 \mathrm{KO}(1.38)$ than WT (1.05) mice, although the difference was not statistically significant (Figure $5 \mathrm{C} ; P>0.05$ ).

The disease burden (total number of histopathological changes in a body) is a good index of tissue and cell injury, which increases with age. Importantly, long-lived mice (e.g., calorie-restricted, Ames, and GHRKO mice) attenuate its age-related increase [20, 22, 23]. The disease burden was slightly less in $\operatorname{Tg}(T X N 2)^{+/ 0} \mathrm{x} \operatorname{Trx} 1 \mathrm{KO}(3.67)$ than WT (4.15) mice. However, these differences are not statistically significant (Figure 5D; $P>0.05$ ).

\section{Discussion}

Thioredoxin (Trx) was initially discovered in the early 1960s as the reductant for a variety of enzymes, and many studies demonstrated its unique roles in biology and physiology [6]. Trx is a hydrogen donor involved in reductive reactions of enzymes (e.g., ribonucleotide reductase, peroxiredoxin (Prx), and methionine sulfoxide (MetO) [7-12], 


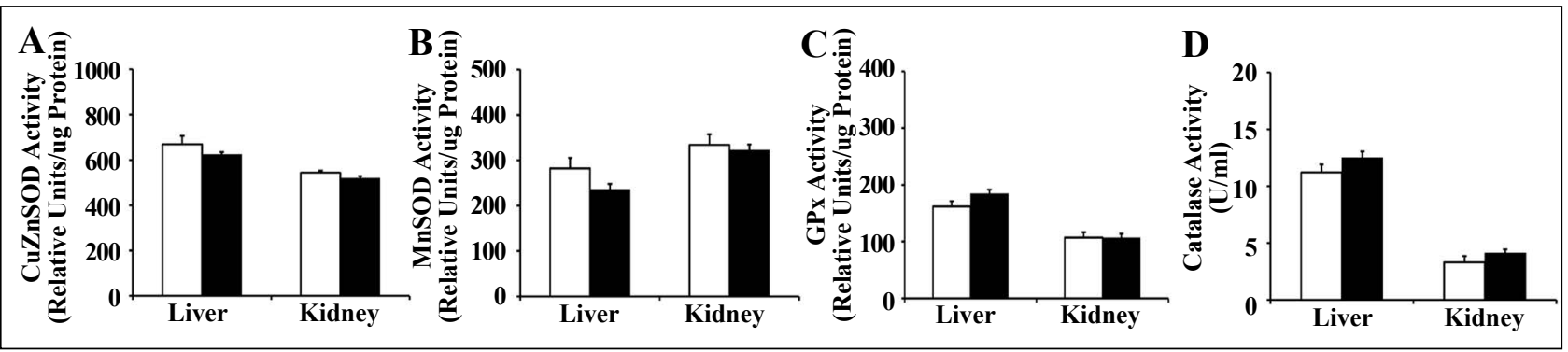

Figure 3. Cu/ZnSOD, MnSOD, GPx, and catalase activity in $\operatorname{Tg}(T X N 2)^{+/ 0} \times \operatorname{Trx} 1 \mathrm{KO}$ and WT mice. The activities of Cu/ZnSOD (A), MnSOD (B), GPx (C), and catalase (D) were measured in the liver and kidney of 4 to 6 month old $\operatorname{Tg}(T X N 2)^{+/ 0}$ x Trx $1 \mathrm{KO}$ (closed bar) and WT (open bar) mice. Cu/ ZnSOD, MnSOD, GPx, and catalase activities were similar between $\operatorname{Tg}(T X N 2)^{+/ 0} \mathrm{x}$ Trx1KO and WT mice. Data are the mean SEM of three mice.

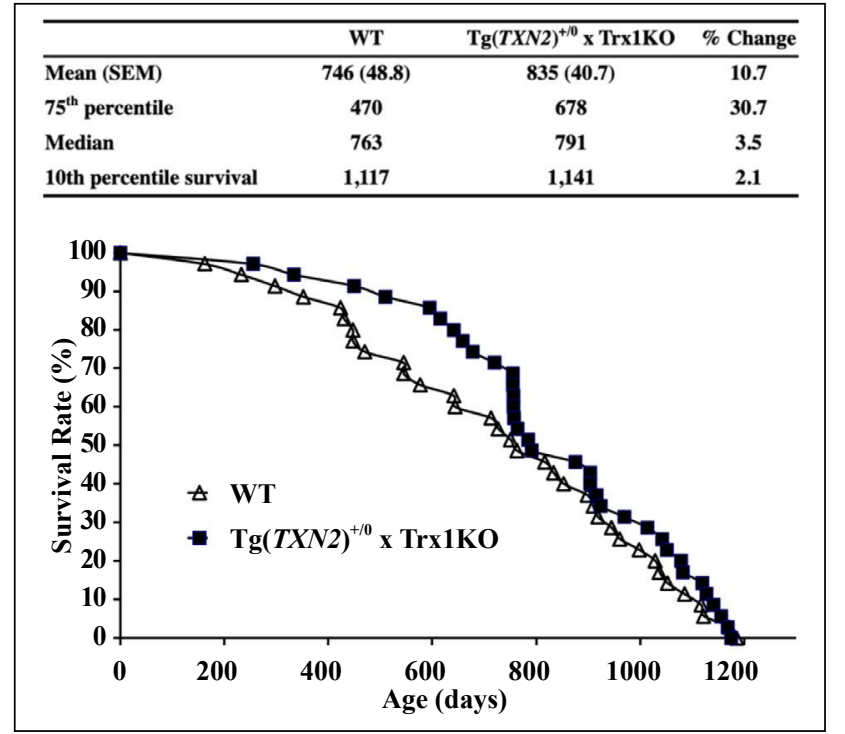

Figure 4. Survival curves of $\operatorname{Tg}(T X N 2)^{+/ 0} \times \operatorname{Trx} 1 \mathrm{KO}$ and WT mice. The survival curves of $\operatorname{Tg}(T X N 2)^{+/ 0} \times \operatorname{Trx} 1 \mathrm{KO}$ (closed squares) and WT (open triangles) mice are presented. The cohort consists of $35 \operatorname{Tg}(T X N 2)^{+/ 0}$ $\mathrm{x} \operatorname{Trx} 1 \mathrm{KO}$ mice and $35 \mathrm{WT}$ male mice. Although $\operatorname{Tg}(T X N 2)^{+/ 0} \mathrm{x} \operatorname{Trx} 1 \mathrm{KO}$ mice slightly extended the lifespan in the early stage of life $(30.7 \%$ extension at 75 th percentile), the survival curves were not significantly different between $\operatorname{Tg}(T X N 2)^{+/ 0} \mathrm{x} \operatorname{Tr} \times 1 \mathrm{KO}$ and WT mice.

which play important roles in biology and cellular function. Trx also plays a key role in maintaining a reduced cellular environment, which provides protection against oxidative stress and control signaling pathways [24-27]. Trx mainly localizes in the cytosol (hTrx1) [28] and mitochondria (hTrx2) [29] in human cells. Studies with mice null for either Trx 1 or Trx 2 showed that these mice are embryonically lethal, which demonstrated that the presence of Trx 1 and Trx2 is essential for mammalian cells/ tissues [30, 31]. Because substantial evidence indicates Trx and thioredoxin interacting protein (Txnip) regulate essential cellular functions $[6,32]$, there is much scientific interest in how Trx could regulate mammalian aging.

Effects of overexpressed or downregulated Trx in the cytosol and/or mitochondria on aging and age-related diseases have been systemically investigated by our laboratory using transgenic and knockout mice for $\operatorname{Trx} 1$ or Trx2 [1-5, 21]. The first Trx study we conducted involved survival experiments with two lines of Trx 1 transgenic mice $\left[\operatorname{Tg}(\operatorname{act}-T X N)^{+/ 0}\right.$ and $\operatorname{Tg}(T X N)^{+/ 0}$ mice] $[2,3]$. These survival studies demonstrated that overexpression of Trx 1 only showed a slight extension in the earlier part of life, and no significant effects on maximum lifespan were observed, possibly due to accelerated tumor development in the later part of life $[2,3]$.

The next study we conducted was a survival experiment with $\operatorname{Trx} 2$ transgenic mice $\left[\operatorname{Tg}(T X N 2)^{+/ 0}\right]$. This study was conducted because of the strong indication that the protection of the mitochondria from age-related increases in oxidative stress may delay aging. The survival curve for $\operatorname{Tg}(T X N 2)^{+/ 0}$ mice showed a slight (approximately 8-9\%) extension of mean, median, and 10th percentile lifespans compared to WT mice, although the extension of lifespan was not statistically significant. $\operatorname{Tg}(T X N 2)^{+/ 0}$ mice also showed a trend that the severity of lymphoma was slightly higher than WT mice [4].

These studies using Trx 1 and Trx 2 transgenic mice could indicate that overexpression of Trx in only one compartment of the cell may not have a significant impact on aging. Our studies with $\operatorname{Trx} 1$ or Trx 2 knockout (KO) mice also showed down-regulation of Trx only in one compartment of the cell showed little impact on aging [5, 14]. Therefore, we decided to test the combined effects of Trx expression changes in both the cytosol and mitochondria. Among the possible combinations of changes (either up-regulation or down-regulation) of Trx1 and $\operatorname{Trx} 2$, we chose to down-regulate $\operatorname{Trx} 1$ and overexpress $\operatorname{Tr} x 2$ in mitochondria for this study because one of the potential reasons Trx 2 overexpression did not significantly extend lifespan could be due to its effects on age-related tumor formation [4]. Our previous study showed that Trx1 down-regulation (Trx1KO mice) had a subtle reduction of age-related tumor incidence [5]. Therefore, the purpose of this study is to test if reduced Trx 1 along with Trx 2 overexpression could extend the lifespan and attenuate tumor formation in $\operatorname{Tg}(T X N 2)^{+/ 0} \times \operatorname{Trx} 1 \mathrm{KO}$ mice.

To test the effects of reduced Trx 1 along with Trx 2 overexpression on aging and age-related diseases, we generated $\operatorname{Tg}(T X N 2)^{+/ 0} \times \operatorname{Trx} 1 \mathrm{KO}$ mice by crossing male heterozygous Trx1KO mice with female hemizygous Trx2 transgenic mice $\left[\operatorname{Tg}(T X N 2)^{+/ 0}\right]$. These genetic manipulations did not cause changes in food intake, body weight, or organ weight. Young (4-6 months old) male $\operatorname{Tg}(T X N 2)^{+/ 0}$ $x$ Trx $1 K O$ mice showed significantly higher Trx 2 and significantly reduced $\operatorname{Trx} 1$ levels in all the tissues examined 


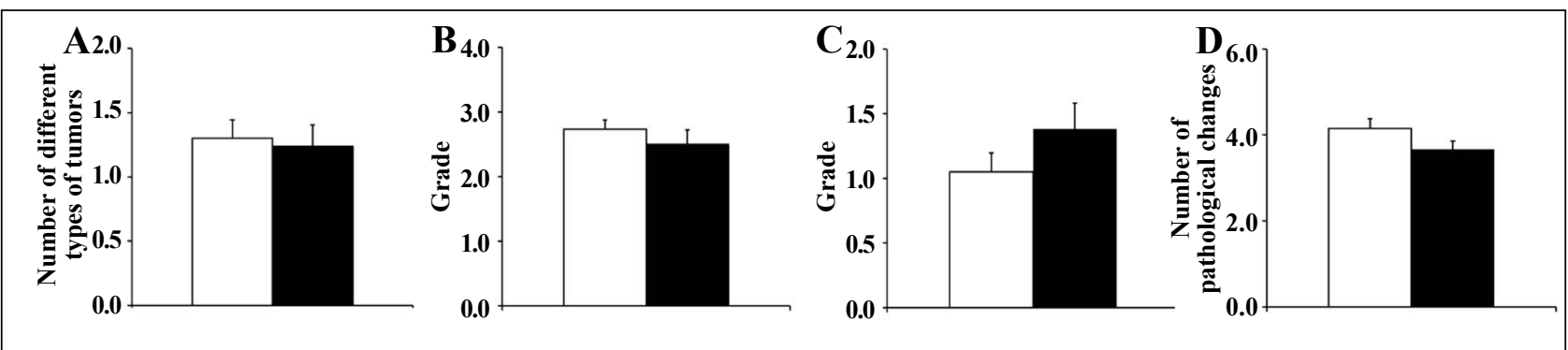

Figure 5. Tumor burden, severity of lymphoma and glomerulonephritis, and disease burden in $\operatorname{Tg}(\operatorname{TXN2})^{+/ 0} \times \operatorname{Trx}_{\mathrm{KOO}}$ and $\mathrm{WT}$ mice. Tumor burden (the number of different types of tumors) (Figure 5A), the severity of lymphoma (Figure 5B), severity of glomerulonephritis (Figure 5C), and disease burden (Figure 5D) in $\operatorname{Tg}(T X N 2)^{+/ 0} \mathrm{x}$ Trx1KO (closed bar) and WT (open bar) mice were compared. The tumor burden and severity of lymphoma for the $\operatorname{Tg}(T X N 2)^{+/ 0} \mathrm{x}$ Trx $1 \mathrm{KO}$ mice were similar to WT mice $(P>0.05)$. Severity of glomerulonephritis was slightly higher in Tg $(T X N 2)^{+/ 0}$ $\mathrm{x}$ Trx $1 \mathrm{KO}$ than WT mice, however, this difference was not statistically significant $(P>0.05)$. The disease burden was slightly less in Tg $(T X N 2)^{+/ 0} \mathrm{x}$ Trx1KO (3.67) than WT (4.15) mice, which also did not reach statistical significance.

compared to WT control mice. The Trx 2 and Trx 1 levels were 1.5 - to 3 -fold higher and approximately $50 \%$ less in all of the tissues examined, respectively. The changes in $\operatorname{Trx} 2$ and $\operatorname{Trx} 1$ levels in male $\operatorname{Tg}(T X N 2)^{+/ 0} \mathrm{x} \operatorname{Trx} 1 \mathrm{KO}$ mice did not cause changes in total glutathione levels or the activities of major antioxidant enzymes $(\mathrm{Cu} / \mathrm{ZnSOD}, \mathrm{Mn}$ SOD, GPx, and catalase).

Our survival study demonstrated that the survival curve of male $\operatorname{Tg}(T X N 2)^{+/ 0} \mathrm{x} \operatorname{Trx} 1 \mathrm{KO}$ mice was not significantly different from WT control mice. Interestingly, the $\operatorname{Tg}(T X N 2)^{+/ 0} \times \operatorname{Tr} \times 1 \mathrm{KO}$ mice showed a slight extension of lifespan in the early stage of life, i.e., 75th percentile lifespan of $\operatorname{Tg}(T X N 2)^{+/ 0} \mathrm{x}$ Trx $1 \mathrm{KO}$ mice was $30.7 \%$ longer than WT mice although these changes were not statistically significant. Mean, median, and 10th percentile lifespans of $\operatorname{Tg}(T X N 2)^{+/ 0} \times \operatorname{Trx} 1 \mathrm{KO}$ mice were similar to WT control mice. The cross-sectional pathology data showed that both $\operatorname{Tg}(T X N 2)^{+/ 0} \times \operatorname{Trx} 1 \mathrm{KO}$ and WT mice had a similar number of total tumors (tumor burden) $\left[\operatorname{Tg}(T X N 2)^{+/ 0} \mathrm{x} \operatorname{Trx} 1 \mathrm{KO}\right.$ mice: 1.24; and WT control mice: 1.30$]$. The incidence of lymphoma was approximately $10 \%$ less in $\operatorname{Tg}(T X N 2)^{+/ 0} \mathrm{x}$ Trx1KO than WT control mice, however, the severity of lymphoma was similar between $\operatorname{Tg}(T X N 2)^{+/ 0} \mathrm{x} \operatorname{Trx} 1 \mathrm{KO}$ and WT mice. In addition to neoplastic diseases, the total number of pathological changes (disease burden) was also similar between $\operatorname{Tg}(T X N 2)^{+/ 0} \mathrm{x} \operatorname{Trx} 1 \mathrm{KO}$ and WT control mice. The severity of glomerulonephritis, which is one of the major non-neoplastic lesions, was slightly higher in $\operatorname{Tg}(T X N 2)^{+/ 0} \mathrm{x}$ Trx $1 \mathrm{KO}$ than WT control mice (not statistically significant). These pathological observations indicate that down-regulation of $\operatorname{Tr} x 1$ along with $\operatorname{Tr} x 2$ overexpression had little effect on age-related pathology, although the $\operatorname{Tg}(T X N 2)^{+/ 0} \times \operatorname{Trx} 1 \mathrm{KO}$ mice slightly extended the earlier stage of lifespan (75th percentile) compared to WT control mice.

The outcome of this study clearly showed that $\operatorname{Trx} 1$ down-regulation combined with Trx2 overexpression did not support our working hypothesis, i.e., $\operatorname{Tg}(T X N 2)^{+/ 0} \mathrm{x}$ Trx1KO mice did not significantly extend lifespan and attenuate age-related diseases. To examine the synergistic effects of $\operatorname{Trx} 1$ and $\operatorname{Trx} 2$, we also conducted a survival study with mice overexpressing Trx1 and Trx2 [1]. This study demonstrated that overexpression of Trx in both the cytosol and mitochondria shortened the lifespan and accelerated cancer development in male C57BL/6 mice, which was contrary to our expectations [1]. Furthermore, the preliminary data from our current ongoing study show that reduced thioredoxin levels in both the cytosol and mitochondria [Trx $1 \mathrm{KO} \times \operatorname{Tr} x 2 \mathrm{KO}$ mice] slightly extend the lifespan in both male and female C57BL/6 mice, which is accompanied by suppressed cancer formation [5]. This study, along with the studies involving either overexpressing or down-regulating Trx 1 or Trx 2 strongly suggest that synergetic overexpression or down-regulation of Trx 1 and $\operatorname{Tr} x 2$ may be required to have significant effects on lifespan and age-related pathology, specifically cancer.

\section{Declarations}

Acknowledgments: We acknowledge the Pathology Core in the San Antonio Nathan Shock Center (P30-AG013319) for pathological analyses and funded by the VA Merit Review grant from the Department of Veterans Affairs.

Conflict of interest: Yuji Ikeno is a member of the Editorial Board of Aging Pathobiology and Therapeutics.All authors declare no conflict of interest and were not involved in the journal's review or desicions related to this manuscript.

\section{References}

1. Cunningham G M, Flores L C, Roman M G, et al. Thioredoxin overexpression in both the cytosol and mitochondria accelerates age-related disease and shortens lifespan in male C57BL/6 mice. Geroscience, 2018, 40(5-6): 453-468.

2. Flores L C, Roman M G, Cunningham G M, et al. Continuous overexpression of thioredoxin 1 enhances cancer development and does not extend maximum lifespan in male C57BL/6 mice. Pathobiology of Aging \& AgeRelated Diseases, 2018, 8(1): 1533754.

3. Pérez V I, Cortez L A, Lew C M, et al. Thioredoxin 1 overexpression extends mainly the earlier part of life span in mice. Journals of Gerontology Series A: Biomedical Sciences and Medical Sciences, 2011, 66(12): 1286-1299.

4. Roman M G, Flores L C, Cunningham G M, et al. Thiore- 
doxin overexpression in mitochondria showed minimum effects on aging and age-related diseases in male C57BL/6 mice. Aging Pathobiology and Therapeutics, 2020, 2(1): 20-31.

5. Roman M G, Flores L C, Cunningham G M, et al. Thioredoxin and aging: What have we learned from the survival studies?. Aging Pathobiology and Therapeutics, 2020, 2(3): 126-133.

6. Arnér E S J, Holmgren A. Physiological functions of thioredoxin and thioredoxin reductase. European Journal of Biochemistry, 2000, 267(20): 6102-6109.

7. Brot N, Weissbach L, Werth J, et al. Enzymatic reduction of protein-bound methionine sulfoxide. Proceedings of the National Academy of Sciences, 1981, 78(4): 2155-2158.

8. Brot N, Weissbach H. Peptide methionine sulfoxide reductase: biochemistry and physiological role. Peptide Science, 2000, 55(4): 288-296.

9. Chae H Z, Kang S W, Rhee S G. Isoforms of mammalian peroxiredoxin that reduce peroxides in presence of thioredoxin. Methods in enzymology, 1999, 300: 219-226.

10. Chae H Z, Kim H J, Kang S W, et al. Characterization of three isoforms of mammalian peroxiredoxin that reduce peroxides in the presence of thioredoxin. Diabetes research and clinical practice, 1999, 45(2-3): 101-112.

11. Kim K, Kim I H, Lee K Y, et al. The isolation and purification of a specific" protector" protein which inhibits enzyme inactivation by a thiol/Fe (III)/02 mixed-function oxidation system. Journal of Biological Chemistry, 1988, 263(10): 4704-4711.

12. Levine R L, Berlett B S, Moskovitz J, et al. Methionine residues may protect proteins from critical oxidative damage. Mechanisms of ageing and development, 1999, 107(3): 323-332.

13. Schriner S E, Linford N J, Martin G M, et al. Extension of murine life span by overexpression of catalase targeted to mitochondria. science, 2005, 308(5730): 1909-1911.

14. Pérez V I, Lew C M, Cortez L A, et al. Thioredoxin 2 haploinsufficiency in mice results in impaired mitochondrial function and increased oxidative stress. Free Radical Biology and Medicine, 2008, 44(5): 882-892.

15. Sun Y, Elwell J H, Oberley L W. A simultaneous visualization of the antioxidant enzymes glutathione peroxidase and catalase on polyacrylamide gels. Free radical research communications, 1988, 5(2): 67-75.

16. Beauchamp C, Fridovich I. Superoxide dismutase: improved assays and an assay applicable to acrylamide gels. Analytical biochemistry, 1971, 44(1): 276-287.

17. Williams M D, Van Remmen H, Conrad C C, et al. Increased oxidative damage is correlated to altered mitochondrial function in heterozygous manganese superoxide dismutase knockout mice. Journal of Biological Chemistry, 1998, 273(43): 28510-28515.

18. Andersen P K, Borgan O, Gill R D, et al. Statistical models based on counting processes. Springer Science \& Business Media, 2012.
19. Wang C, Li Q, Redden D T, et al. Statistical methods for testing effects on "maximum lifespan". Mechanisms of ageing and development, 2004, 125(9): 629-632.

20. Bronson R T, Lipman R D. of Age Related Lesions in Dietary Restricted Laboratory Mice. Growth, Development \& Aging, 1991, 55: 169-184.

21. Ikeno Y, Hubbard G B, Lee S, et al. Housing density does not influence the longevity effect of calorie restriction. The Journals of Gerontology Series A: Biological Sciences and Medical Sciences, 2005, 60(12): 1510-1517.

22. Ikeno Y, Bronson R T, Hubbard G B, et al. Delayed occurrence of fatal neoplastic diseases in Ames dwarf mice: correlation to extended longevity. The Journals of Gerontology Series A: Biological Sciences and Medical Sciences, 2003, 58(4): B291-B296.

23. Ikeno Y, Hubbard G B, Lee $S$, et al. Reduced incidence and delayed occurrence of fatal neoplastic diseases in growth hormone receptor/binding protein knockout mice. Journals of Gerontology Series A: Biomedical Sciences and Medical Sciences, 2009, 64(5): 522-529.

24. Abate C, Patel L, Rauscher F J, et al. Redox regulation of fos and jun DNA-binding activity in vitro. Science, 1990, 249(4973): 1157-1161.

25. Galter D, Mihm S, DRÖGE W. Distinct effects of glutathione disulphide on the nuclear transcription factors $\kappa B$ and the activator protein-1. European Journal of Biochemistry, 1994, 221(2): 639-648.

26. Takagi Y, Mitsui A, Nishiyama A, et al. Overexpression of thioredoxin in transgenic mice attenuates focal ischemic brain damage. Proceedings of the National Academy of Sciences, 1999, 96(7): 4131-4136.

27. Toledano M B, Leonard W J. Modulation of transcription factor NF-kappa B binding activity by oxidationreduction in vitro. Proceedings of the National Academy of Sciences, 1991, 88(10): 4328-4332.

28. Tagaya Y, Maeda Y, Mitsui A, et al. ATL-derived factor (ADF), an IL-2 receptor/Tac inducer homologous to thioredoxin; possible involvement of dithiol-reduction in the IL-2 receptor induction. The EMBO journal, 1989, 8(3): 757-764.

29. Spyrou G, Enmark E, Miranda-Vizuete A, et al. Cloning and expression of a novel mammalian thioredoxin. Journal of Biological Chemistry, 1997, 272(5): 2936-2941.

30. Matsui M, Oshima M, Oshima H, et al. Early embryonic lethality caused by targeted disruption of the mouse thioredoxin gene. Developmental biology, 1996, 178(1): 179-185.

31. Nonn L, Williams R R, Erickson R P, et al. The absence of mitochondrial thioredoxin 2 causes massive apoptosis, exencephaly, and early embryonic lethality in homozygous mice. Molecular and cellular biology, 2003, 23(3): 916-922.

32. Yoshihara E, Masaki S, Matsuo Y, et al. Thioredoxin/ Txnip: redoxisome, as a redox switch for the pathogenesis of diseases. Frontiers in immunology, 2014, 4: 514.

Cite this article as: Roman M G, Flores L C, Cunningham G M, et al. Thioredoxin down-regulation in the cytosol in thioredoxin 2 transgenic mice did not have beneficial effects to extend lifespan in male C57BL/6 mice[J]. Aging Pathobiology and Therapeutics, 2020, 2(4): 203-209. 